\title{
Clinical Safety of a High Dose of Phycocyanin-Enriched Aqueous Extract from Arthrospira (Spirulina) platensis: Results from a Randomized, Double-Blind, Placebo-Controlled Study with a Focus on Anticoagulant Activity and Platelet Activation
}

\author{
Gitte S. Jensen, ${ }^{1}$ Cassandra Drapeau, ${ }^{2}$ Miki Lenninger, ${ }^{1}$ and Kathleen F. Benson ${ }^{1}$ \\ ${ }^{I}$ NIS Labs, Klamath Falls, Oregon, USA. \\ ${ }^{2}$ Cerule LLC, Klamath Falls, Oregon, USA.
}

\begin{abstract}
The goal for this study was to evaluate safety regarding anticoagulant activity and platelet activation during daily consumption of an aqueous cyanophyta extract (ACE), containing a high dose of phycocyanin. Using a randomized, double-blind, placebo-controlled study design, 24 men and women were enrolled after informed consent, and consumed either ACE (2.3 g/day) or placebo daily for 2 weeks. The ACE dose was equivalent to $\sim 1 \mathrm{~g}$ phycocyanin per day, chosen based on the highest dose Generally Recognized as Safe (GRAS) by the U.S. Food and Drug Administration. Consuming ACE did not alter markers for platelet activation (P-selectin expression) or serum P-selectin levels. No changes were seen for activated partial thromboplastin time, thrombin clotting time, or fibrinogen activity. Serum levels of aspartate transaminase (AST) showed a significant reduction after 2 weeks of ACE consumption $(P<.001)$, in contrast to placebo where no changes were seen; the difference in AST levels between the two groups was significant at 2 weeks $(P<.02)$. Reduced levels of alanine transaminase (ALT) were also seen in the group consuming ACE $(P<.08)$. Previous studies showed reduction of chronic pain when consuming $1 \mathrm{~g}$ ACE per day. The higher dose of $2.3 \mathrm{~g}$ /day in this study was associated with significant reduction of chronic pain at rest and when physically active $(P<.05)$. Consumption of ACE showed safety regarding markers pertaining to anticoagulant activity and platelet activation status, in conjunction with rapid and robust relief of chronic pain. Reduction in AST and ALT suggested improvement in liver function and metabolism.
\end{abstract}

KEY WORDS: • anticoagulant activity $\bullet$ blood pressure $\bullet$ chronic pain $\bullet$ liver enzymes $\bullet$ platelet $\bullet P-$ selectin

\section{INTRODUCTION}

C YANOBACTERIA, OR BLUE-GREEN ALGAE, have survived and evolved over the last 3.5 billion years and are considered the evolutionary bridge between bacteria and green plants. Their adaptive ability to harvest sunlight through a broader light spectrum than most green plants, as well as their ability to utilize other forms of energy, has made these organisms well adapted to survive and inhabit diverse habitats hostile to other life forms. ${ }^{1,2}$ Edible cyanobacteria, including Spirulina platensis, Spirulina maxima, and Aphanizomenon flos-aquae, were introduced to the health food market in the late 1970s and have gained considerable popularity in the health food industry. The bright blue light-harvesting pigment phycocyanin, unique to cyanobacteria, is a known an-

Manuscript received 23 November 2015. Revision accepted 20 May 2016.

Address correspondence to: Gitte S. Jensen, PhD, NIS Labs, 1437 Esplanade, Klamath Falls, OR 97601, USA, E-mail: gitte@nislabs.com tioxidant and anti-inflammatory compound, in part, due to its ability to inhibit the inflammatory enzyme, cyclooxygenase$2(\mathrm{COX}-2) .^{3-5}$

Pharmaceutical inhibitors of COX enzymes, nonsteroidal anti-inflammatory drugs (NSAIDs), are commonly used anti-inflammatory medications for pain relief; however, their use is associated with side effects, including gastrointestinal discomfort and mucosal ulceration, ${ }^{6}$ and longterm use poses a risk for kidney disease especially in the hypertensive population. ${ }^{7}$ In contrast to the acute damage associated with NSAID intake, anti-inflammatory effects seem to provide a mild long-term benefit in the prevention of colorectal cancer. ${ }^{8}$ Some NSAIDs such as aspirin and ibuprofen, both nonspecific COX inhibitors, also have anticoagulant effects, which depending on dosage can be used prophylactically in the prevention of coagulopathy, ${ }^{9}$ or at higher doses are used for reducing pain and inflammation but can contribute significant gastrointestinal risks. ${ }^{6}$ Furthermore, conflicting evidence exists regarding whether the 
intake of NSAIDs is beneficial or detrimental to different types of bone- and joint-related diseases. ${ }^{10}$ An association has been suggested between intake of aspirin and the prevention of the progression of nontraumatic necrotic bone disease in young adults. ${ }^{11}$ In contrast, intake of NSAIDs in adults with different types of arthritis has been associated with negative impact on bone metabolism. ${ }^{12,13}$ Recent research warns that certain NSAID medications affect the gut-brain axis, triggering broad systemic, endocrine, and behavioral changes, including disturbances in heart rate, body temperature, and anxiety-like behavior. ${ }^{14}$

Seeking nonpharmaceutical interventions for chronic pain has led to an increasing attractiveness of natural products with COX-2 inhibiting properties. ${ }^{15-17}$ Spirulina's safety as a food has been established through centuries of human use, as well as through numerous toxicology studies. ${ }^{18,19}$ However, such safety data do not automatically vouch for the safety of extracts and purified compounds from Spirulina, and hence, further safety documentation is needed for such extracts.

There is a potential for natural compounds to provide more multifaceted biological effects. As an example, phycocyanin's bioactivity extends beyond its COX-2 inhibiting properties. Phycocyanin has been shown to activate Nrf2, ${ }^{20}$ and this may account for its effect on heme oxygenase-1 (HO-1). ${ }^{21}$ The activation of HO-1 may be secondary to Nrf2 activation, and Nrf2 activation could also contribute to activation of phase II enzymes in the liver and decreased liver enzymes. ${ }^{22}$ Phycocyanin contains an open-chain tetrapyrrole chromophore known as phycocyanobilin (PCB), with a chemical structure similar to that of biliverdin, susceptible to biliverdin reductase, giving rise to phycocyanorubin, an analog of bilirubin. ${ }^{23}$ PCB constitutes up to $1 \%$ of the dry weight of Spirulina, with higher free radical scavenging properties than intact phycocyanin, ${ }^{24}$ and strong protective properties in ischemia-induced inflammation, ${ }^{25}$ as well as in diabetic nephropathy. ${ }^{26}$ Consumed PCB may have a considerable potential for preventing or slowing the progression of a wide range of neurodegenerative disorders. ${ }^{27}$ These bioactivities of PCB may, in part, be explained by its potent inhibition of NADPH oxidase, ${ }^{23}$ which plays a key role in the generation of superoxide during the respiratory burst in neutrophils. Due to the inflammatory generation of free radicals by NADPH oxidase, it is a major cause of atherosclerosis, and NADPH oxidase inhibitors may reverse atherosclerosis. ${ }^{28}$ PCB also activates atheroprotective heme oxygenase-1 (HMOX1) in endothelial cells, and its multifaceted effects may represent an important mechanism of this food supplement for the reduction of atherosclerotic disease. ${ }^{29}$ The ability of HO-1 to catabolize free heme and produce carbon monoxide (CO) suggests that its antiinflammatory properties involve upregulation of IL-10 and IL-1R antagonist expression. ${ }^{30}$

Specifically, due to the COX-2 inhibiting properties of phycocyanin, it may potentially interfere with blood clotting, at least when pure phycocyanin is introduced to molecular and cellular components of the blood clotting machinery in vitro. Purified phycocyanin from Spirulina fusiformis was tested for its effect on the fibrinolytic system in vascular endothelial cells in vitro, where phycocyanin increased the fibrinolytic activity in a dose-dependent manner. ${ }^{31}$ Purified phycocyanin is used for injection in medical imaging due to its fluorescent properties, and injectable phycocyanin may represent a potential pharmaceutical agent for the treatment of thrombosis due to its potent effects on multiple aspects of platelet activation, causing a reduction of platelet aggregation at nanomolar concentrations. ${ }^{32,33}$ In contrast, using the euglobulin fibrinolytic assay, we have previously reported that phycocyanin did not inhibit blood clotting in vitro, where clot formation and subsequent fibrinolysis were monitored in the absence versus presence of phycocyanin. Our data showed that phycocyanin contributed to the antioxidant properties and anti-inflammatory effects, without a negative impact on blood clotting in vitro; the time for clot formation and lysis was not affected. ${ }^{34}$

Whether the consumption of phycocyanin results in a measurable effect on components of the blood clotting molecular machinery in humans remained a pertinent safety question, and was the motivation for conducting the study reported here. Previous pilot studies on the phycocyanin-rich aqueous cyanophyta extract (ACE) used for the current study showed that the consumption of ACE at a dose of $0.25-1.0 \mathrm{~g} /$ day was associated with relief of chronic pain. ${ }^{35}$ In light of conflicting data from laboratory tests and the question of whether consumed phycocyanin could lead to anticoagulant effects in vivo, the goal for the study reported here was to document important safety parameters specifically pertaining to blood coagulation and platelet activation in a human population when ingesting a higher dose of $2.3 \mathrm{~g}$ ACE per day, corresponding to $\sim 1 \mathrm{~g}$ of phycocyanin per day.

\section{MATERIALS AND METHODS}

\section{Study design}

A randomized, double-blind, placebo-controlled study design was used for this clinical study. Twenty-four people qualified for enrollment in the 2-week study on signing written informed consent, as approved by the Sky Lakes Institutional Review Board. The inclusion and exclusion criteria are shown in Table 1. The participants were randomized to receive either product or placebo for the 2week study. The prescreening involved an interview to document gender, age, body mass index (BMI), medical/ surgical history, diet/lifestyle, current health issues, medication, and supplement use. If subjects met these criteria, they were scheduled for a screening visit to evaluate electrocardiogram (ECG) and blood chemistry. Subjects who passed the prescreening interview and met the screening criteria were invited to participate and after signing an informed consent were enrolled in the study. The study was carried out during 2014/2015 through NIS Labs located in southern Oregon (United States), where study participants live and work at an elevation of $1200-1500 \mathrm{~m}$ above sea level. 
Table 1. Inclusion and Exclusion Criteria for the Study

Inclusion criteria

Adult men and women $25-65$ years of age;

BMI below 35;

Chronic pain related to specific joint(s), for more than 6 months.

Exclusion criteria

Currently taking medication for blood pressure or lipidemia;

Currently taking anti-inflammatory nutritional supplements judged by the study coordinator to camouflage or enhance the effects of the test product;

Unwilling to maintain a constant intake of supplements over the duration of the study;

Currently taking prednisone, or regular prednisone consumption within last 3 months;

Known active liver disease;

Known active kidney disease;

Known active cardiovascular health issues;

Known active inflammatory or autoimmune disease;

Cancer and/or chemotherapy during last year;

Family history of sudden death before the age of 40 ;

Currently experiencing intense stressful events/life changes that could affect compliance;

Food allergies related to test product.

BMI, body mass index.

\section{Consumable test products}

The active consumable product for this study is an ACE, Cyactiv $^{\circledR}$, and is based on proprietary aqueous processing of Arthrospira platensis biomass, produced at Cerule LLC (Klamath Falls, OR, USA). The lot used for this study was obtained from Cerule LLC, where the extract Cyactiv is produced using a proprietary process involving aqueous extraction technology. The lot had $\sim 40 \%$ phycocyanin by weight, and also contained nonphycocyanin bioactive antiinflammatory compounds. ${ }^{31} \mathrm{~A}$ color-matched placebo powder was made from rice flour. The ACE and placebo were encapsulated in vegetarian cellulose capsules and bottled in similar bottles. Subjects were instructed to return bottles with unused capsules at the study exit visit, and remaining capsules were used to document compliance.

\section{Blood pressure}

Blood pressure readings were performed using an Omron 741 or 742 monitor, and were scheduled at the same time of the day for each subject. At each study visit, blood pressure was measured two or three times at a sitting position after a 5-min rest, with at least a 3-min interval between each measurement. Subjects were asked not to smoke, eat, or exercise $1 \mathrm{~h}$ before their appointment, and all study participants adhered to the requirements of abstinence from exercise and nicotine and caffeine use for $1 \mathrm{~h}$ before a study visit.

\section{Electrocardiogram}

At screening and the 2-week follow-up, a three-lead ECG was performed.

\section{Blood collection}

On each of the two study visits (baseline, 2 weeks), a blood sample was drawn. A complete blood count with differential count was performed, as well as blood chemistry. Additional serum samples were used for testing for activated partial thromboplastin time (aPTT), thrombin time (TT), and fibrinogen activity.

\section{Platelet P-selectin expression}

The level of platelet activation was evaluated by dual staining with the platelet marker CD42a (glycoprotein IX, which together with glycoprotein $\mathrm{Ib}$, forms the receptor for von Willebrand factor) and CD62P. The CD62P selectin is a selectin-type cell adhesion molecule, residing in intraplatelet vacuoles until platelets are activated, after which CD62P is rapidly expressed on the platelet surface. Whole blood samples were stained with fluorescent anti-CD42a and anti-CD62P monoclonal antibodies (BD Biosciences, San Jose, CA, USA) and incubated for $20 \mathrm{~min}$ in the dark. Red blood cells were lysed using High-Yield Lyse (Life Technologies, Grand Island, NY, USA), vortexed, and incubated for $10 \mathrm{~min}$. All samples were stained and analyzed in triplicate. Samples were acquired within $2 \mathrm{~h}$ using the acoustic aligning Attune ${ }^{\circledR}$ flow cytometer. Platelets and plateletbound leukocytes were identified as CD42a-positive particles, and electronic gating on CD42a-positive particles was used to analyze platelets for CD62P fluorescence intensity.

\section{Soluble P-selectin}

Serum samples were tested in duplicate for soluble Pselectin using a Luminex magnetic bead-based kit (ProcartaPlex Human P-Selectin Simplex; Ebioscience, San Diego, CA, USA), performed according to the manufacturer's instructions. Samples were analyzed on the MAGPIX instrument (Luminex Corporation, Austin, TX, USA) using the XPONENT software (Version 4.2; Software Solution for Luminex, Hoorn, The Netherlands).

\section{Coagulation markers}

Plasma samples were tested for aPTT, TT, and fibrinogen activity at Machaon Diagnostic Laboratory (Oakland, CA, USA).

\section{Pain assessment}

The pain assessments at the baseline and the 2-week visits involved the scoring of pain levels in the areas of the body identified by each study participant as their primary and secondary area of pain limiting their daily activities. Scoring was performed using the visual analogue scales (VAS, 0-100), and subjects were asked to score their individual pain areas for pain levels when at rest and when physically active.

\section{Statistical analysis}

Average and standard deviation for each data set was calculated using Microsoft Excel. Statistical analysis of clinical 
Table 2. Demographics of Study Population

\begin{tabular}{|c|c|c|c|}
\hline & Placebo & Cyactiv & $\mathrm{P}$ \\
\hline Females & 9 & 10 & \\
\hline Age $_{\text {average }} \mathrm{a}^{\mathrm{a}}$ & $42.7 \pm 10.5$ & $50.1 \pm 11.5$ & .1608 \\
\hline Age range & $25.6-53.6$ & $25.7-65.9$ & \\
\hline BMI average ${ }^{a}$ & $26.9 \pm 4.8$ & $29 \pm 3.1$ & .3008 \\
\hline BMI range & $20.5-33.9$ & $24.5-33.5$ & \\
\hline Males & 3 & 2 & \\
\hline Age average $\mathrm{e}^{\mathrm{a}}$ & $44.1 \pm 18.6$ & $45.3 \pm 1.2$ & .9189 \\
\hline Age range & $32.1-65.5$ & $44.5-46.1$ & \\
\hline BMI average ${ }^{a}$ & $32.4 \pm 1.9$ & $30.3 \pm 2.8$ & .4697 \\
\hline BMI range & $31-34.6$ & $28.4-32.3$ & \\
\hline
\end{tabular}

${ }^{a}$ The average \pm standard deviation is shown.

data was performed as "between-group" comparison using the two-tailed, unpaired $t$-test. Statistical significance of changes from baseline to the 2-week assessment was evaluated by "within-subject" analysis using the two-tailed paired $t$-test. A trend was defined as $P<.1$. Statistical significance was defined as $P<.05$, and a high level of significance $P<.01$.

\section{RESULTS}

\section{Demographics and compliance}

The study population was predominantly female, with 19 women and 5 men distributed evenly between the placebo and $\mathrm{ACE}$ groups with no significant differences between the age and BMI between the two groups (Table 2). All subjects showed greater than $80 \%$ compliance, with an average compliance of $97 \%$.

\section{Blood pressure}

There were no statistically significant differences between the two groups regarding systolic and diastolic blood pressure at baseline or after 2 weeks. The consumption of ACE was associated with a mild reduction in diastolic blood pressure, but did not reach statistical significance within the 2 -week study $(P<.15)$. This effect was seen for both female and male study participants (Table 3 ).

\section{Electrocardiogram}

All study participants were screened to ensure a normal ECG before enrolling into the study. There were no changes in individual ECG readings after the 2-week study participation (data not shown).

\section{Blood chemistry}

The blood chemistry data showed a mild, but highly significant reduction in aspartate transaminase (AST) after 2 weeks of ACE consumption $(P<.001)$, in contrast to placebo where no changes were seen; the difference in AST levels between the two groups was significant at 2 weeks $(P<.02)$ (Fig. 1A). In addition, a reduction in alanine transaminase (ALT) was also seen in the group consuming ACE, however, the reduction did not reach statistical significance $(P<.08)$ (Fig. 1B). A mild reduction in the $\mathrm{A} / \mathrm{G}$ ratio was seen in the ACE group. A slight increase in blood $\mathrm{CO}_{2}$ was seen in the ACE group, in contrast to a slight decrease in the placebo group, resulting in a significant difference between the two groups' averages at 2 weeks; however, the changes were minor and well within the normal range. No other changes in blood chemistry were seen (Table 4 ).

\section{Platelets}

The activation status of blood platelets plays an intricate role in blood coagulation. Due to the well-documented COX-2 inhibiting effects of phycocyanin, ${ }^{3-5}$ and therefore a potential role for interfering with platelet function, ${ }^{28-30}$ it was a central part of this study to evaluate various markers pertaining to platelet function and activation status (Fig. 2). During the 2-week study, no changes were seen in platelet numbers in subjects consuming a high dose of $2.3 \mathrm{~g} /$ day of the phycocyanin-enriched extract (Fig. 2A). A small but statistically significant reduction in mean platelet volume was seen in the group consuming ACE $(P<.02)$ (Fig. 2B). No differences were seen between the placebo and ACE groups pertaining to platelet aggregation or platelet Pselectin expression, or serum levels of soluble P-selectin (Fig. 2C, D).

Table 3. Blood Pressure Results

\begin{tabular}{|c|c|c|c|c|c|c|c|}
\hline & Placebo week 0 & Placebo week 2 & $\mathrm{P} *$ & Cyactiv week 0 & Cyactiv week 2 & $\mathrm{P}^{*}$ & $\mathrm{P}^{* *}$ \\
\hline Females & 9 & 9 & 10 & 10 & & & \\
\hline Systolic average $^{\mathrm{a}}$ & $112.7 \pm 8.9$ & $111.3 \pm 9.9$ & .3886 & $120.8 \pm 15.3$ & $120 \pm 15.8$ & .7496 & .7988 \\
\hline Systolic range & $100-127$ & $98-129$ & & 99-155 & $98-148$ & & \\
\hline Diastolic average ${ }^{a}$ & $70.8 \pm 9.1$ & $70.2 \pm 9.6$ & .6402 & $75.5 \pm 12$ & $73.3 \pm 11.6$ & .2017 & .5043 \\
\hline Diastolic range & $56-85$ & $55-81$ & & $58-96$ & $56-94$ & & \\
\hline Males & 3 & 3 & & 2 & 2 & & \\
\hline Systolic average ${ }^{a}$ & $131.7 \pm 5.5$ & $127.7 \pm 9.0$ & .3828 & $113.5 \pm 6.4$ & $114 \pm 2.8$ & .8743 & .3892 \\
\hline Systolic range & $128-138$ & $117-136$ & & $109-118$ & $112-116$ & & \\
\hline Diastolic average $^{a}$ & $75 \pm 7$ & $75.3 \pm 7.6$ & .9333 & $75 \pm 5.7$ & $73.5 \pm 6.4$ & .2048 & .6482 \\
\hline Diastolic range & $70-83$ & $67-82$ & & $71-79$ & $69-78$ & & \\
\hline
\end{tabular}

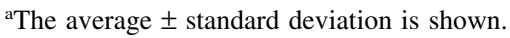

$* P$-values for changes from baseline to 2 weeks within each group.

$* * P$-values for comparing changes between the two groups. 

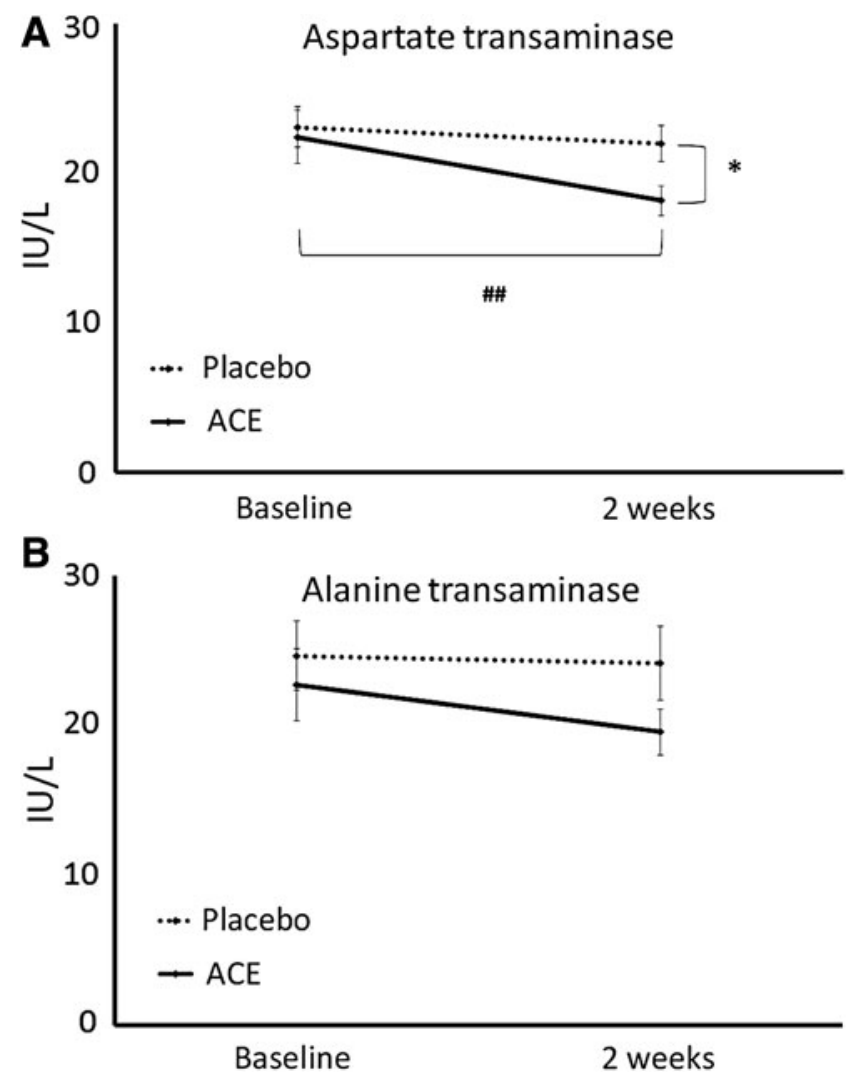

FIG. 1. Serum levels of the two liver enzymes (A) AST and (B) ALT are shown as the group averages \pm standard error of the mean for baseline and 2-week samples from the group consuming the ACE (solid lines) versus placebo (dashed lines). A reduction was seen in the ACE group for both enzymes, where the change for AST reached a high level of statistical significance within the ACE group $\left({ }^{\#} P<.01\right)$. The difference in AST levels between the ACE and placebo groups at 2 weeks was statistically significant $(* P<.02)$. ACE, aqueous cyanophyta extract; ALT, alanine transaminase; AST, aspartate transaminase.

\section{Clotting markers}

Plasma was tested at baseline and after 2 weeks of consuming $2.3 \mathrm{~g} /$ day of the phycocyanin-enriched ACE (Table 5). There were no changes in aPTT or thrombin clotting time between the two groups. There was a mild but statistically insignificant increase in fibrinogen activity in the group consuming $\mathrm{ACE}$, in contrast to the group consuming placebo.

\section{Other observations}

Data were collected regarding subjective observations from study participants, including gastrointestinal discomfort, since this could be a potential concern due to the COX2 inhibiting properties of phycocyanin. There were no reports of gastrointestinal discomfort above what was normal for each study participant. Questions were also asked pertaining to bruising, gum health, and nose bleeds. One person consuming ACE reported mild bruising during the study, and three people consuming ACE, who did not nor- mally notice bleeding gums, reported mild incidents of bleeding gums in association with brushing their teeth during the study.

Female pre-/perimenopausal study participants were asked about symptoms associated with menstrual discomfort as well as bleeding duration and intensity. However, there were only two pre-/perimenopausal female volunteers enrolled in the study, and both volunteers were in the placebo group. Thus, this study did not include systematic data collection on menstrual discomfort or bleeding intensity.

\section{Reduction of chronic pain}

To perform the safety evaluation in a study population that reflects the typical consumer of ACE, the study was conducted in a population with a long history of chronic pain but not diagnosed with a specific joint disease. Based on previous data on pain relief when consuming $1 \mathrm{~g}$ ACE per day, it is not surprising that a robust decrease in pain scores was seen in the ACE group in this study, where study participants were consuming $2.3 \mathrm{~g} / \mathrm{day}$ (Fig. 3). Each individual's anatomical areas for chronic pain were identified before study start, based on the degree with which the pain interfered with daily living. Pain scores were collected for primary and secondary pain complaints at rest and when physically active. Pain scores for primary pain at rest and when active were similar between the two groups at baseline, but were statistically significant after 2 weeks $(P<.05)$. The reduction in primary pain scores within the ACE group was highly significant $(P<.01)$.

\section{DISCUSSION}

The clinical study reported here is the first to address specific safety concerns about consumption of blue-green algal consumables and extracts thereof, where the content of the light-harvesting pigment phycocyanin has raised questions about potential anticoagulation effects in vivo. Edible blue-green algae include Spirulina and Aphanizomenon species and subspecies, containing the COX-2 inhibitor phycocyanin, as well as additional nonphycocyanin antiinflammatory compounds. ${ }^{31}$ Due to the potential of reduced blood coagulation associated with phycocyanin consumption, a previous clinical study collected safety-related data when subjects consumed $1 \mathrm{~g}$ ACE per day for 4 weeks. The results showed no changes in standard safety parameters, including platelet numbers and blood chemistry. ${ }^{32}$ The study presented here involved consumption of $2.3 \mathrm{~g}$ ACE per day for 2 weeks and a comprehensive set of tests specifically pertaining to platelet activation and blood coagulation. The dose of $2.3 \mathrm{~g}$ ACE per day (equivalent to $1 \mathrm{~g}$ phycocyanin) was chosen based on the evaluation by the U.S. Food and Drug Administration where $1 \mathrm{~g} /$ day phycocyanin is Generally Recognized as Safe (GRAS) ${ }^{36}$ Data from this study showed no changes to blood pressure, ECG, blood chemistry, platelet numbers, and activation status as reflected by P-selectin expression. The consumption of ACE did not change aPTT or thrombin clotting time, nor did it reduce normal fibrinogen activity, suggesting that normal blood 
Table 4. Blood Chemistry

\begin{tabular}{|c|c|c|c|c|c|c|c|c|c|}
\hline \multirow[b]{2}{*}{ Test (Unit) } & \multicolumn{2}{|c|}{ Reference ranges } & \multirow{2}{*}{$\begin{array}{c}\text { Placebo } \\
\text { week } 0\end{array}$} & \multirow{2}{*}{$\begin{array}{c}\text { Placebo } \\
\text { week } 2\end{array}$} & \multirow{2}{*}{$\begin{array}{l}\mathrm{P}^{*} \text { placebo } \\
(0-2 \text { weeks })\end{array}$} & \multirow[b]{2}{*}{$A C E$ week $O$} & \multirow[b]{2}{*}{$A C E$ week 2} & \multirow{2}{*}{$\begin{array}{c}\mathrm{P}^{*} A C E \\
(0-2 \text { weeks })\end{array}$} & \multirow{2}{*}{$\begin{array}{c}\mathrm{P} * * \\
\text { placebo } \\
A C E\end{array}$} \\
\hline & Min & $\operatorname{Max}$ & & & & & & & \\
\hline Sodium (mmol/L) & 135.00 & 145.00 & $138.19 \pm 1.16$ & $137.73 \pm 2.11$ & .411 & $137 \pm 2.01$ & $137.39 \pm 1.76$ & .526 & .291 \\
\hline Potassium (mmol/L) & 3.50 & 5.10 & $4.21 \pm 0.25$ & $4.2 \pm 0.22$ & .921 & $4.21 \pm 0.1$ & $4.08 \pm 0.2$ & .027 & .219 \\
\hline Chloride (mmol/L) & 101.00 & 111.00 & $105.56 \pm 1.34$ & $105.64 \pm 1.87$ & .880 & $105.54 \pm 3.02$ & $105.29 \pm 1.65$ & .735 & .757 \\
\hline $\mathrm{CO}_{2}(\mathrm{mmol} / \mathrm{L})$ & 22.00 & 32.00 & $25.68 \pm 1.14$ & $24.86 \pm 2.23$ & .173 & $24.85 \pm 2.32$ & $25.74 \pm 2.34$ & .135 & .038 \\
\hline Calcium (mg/dL) & 8.60 & 10.30 & $9.28 \pm 0.38$ & $9.26 \pm 0.33$ & .882 & $9.1 \pm 0.27$ & $9.02 \pm 0.26$ & .384 & .626 \\
\hline BUN (mg/dL) & 6.00 & 20.00 & $12.58 \pm 3.34$ & $12.08 \pm 2.71$ & .663 & $14.58 \pm 3.58$ & $13.08 \pm 3.26$ & .160 & .422 \\
\hline Creatinine (mg/dL) & 0.60 & 1.30 & $0.83 \pm 0.14$ & $0.81 \pm 0.16$ & .391 & $0.78 \pm 0.19$ & $0.77 \pm 0.14$ & .877 & .375 \\
\hline BUN/Crea ratio & 12.00 & 20.00 & $15.31 \pm 3.83$ & $15.65 \pm 4.97$ & .829 & $19.68 \pm 7.05$ & $17.38 \pm 5.07$ & .169 & .296 \\
\hline Glucose (mg/dL) & 74.00 & 106.00 & $83.5 \pm 11.73$ & $82.08 \pm 8.61$ & .507 & $85.25 \pm 5.99$ & $84.25 \pm 8.5$ & .661 & .983 \\
\hline Total protein $(\mathrm{g} / \mathrm{dL})$ & 6.50 & 8.20 & $6.81 \pm 0.43$ & $6.74 \pm 0.29$ & .572 & $6.75 \pm 0.39$ & $6.74 \pm 0.40$ & .922 & .742 \\
\hline Albumin $(\mathrm{g} / \mathrm{dL})$ & 3.50 & 5.00 & $4.08 \pm 0.4$ & $4.1 \pm 0.27$ & .714 & $3.95 \pm 0.25$ & $3.9 \pm 0.35$ & .515 & .372 \\
\hline $\mathrm{A} / \mathrm{G}$ ratio $^{\mathrm{a}}$ & 1.20 & 2.20 & $1.52 \pm 0.28$ & $1.58 \pm 0.26$ & .104 & $1.46 \pm 0.3$ & $1.43 \pm 0.34$ & .491 & .049 \\
\hline Alk phos (IU/L) & 38.00 & 126.00 & $56.33 \pm 15.74$ & $56.58 \pm 13.53$ & .828 & $62.42 \pm 14.96$ & $62.58 \pm 14.04$ & .892 & .738 \\
\hline Bilirubin (mg/dL) & 0.30 & 1.20 & $0.82 \pm 0.29$ & $0.69 \pm 0.23$ & .068 & $0.77 \pm 0.26$ & $0.78 \pm 0.42$ & .927 & .367 \\
\hline $\mathrm{Ca}$ Osmol (mOsm/kg) & 260.00 & 300.00 & $275.17 \pm 3.17$ & $274.07 \pm 4.19$ & .355 & $273.75 \pm 4.6$ & $273.91 \pm 3.53$ & .909 & .472 \\
\hline
\end{tabular}

The average \pm standard deviation is shown.

${ }^{a}$ Albumin/Globulin ratio.

* $P$-values for changes from baseline to 2 weeks within each group.

** $P$-values for comparing changes between the two groups.

ACE, aqueous cyanophyta extract.
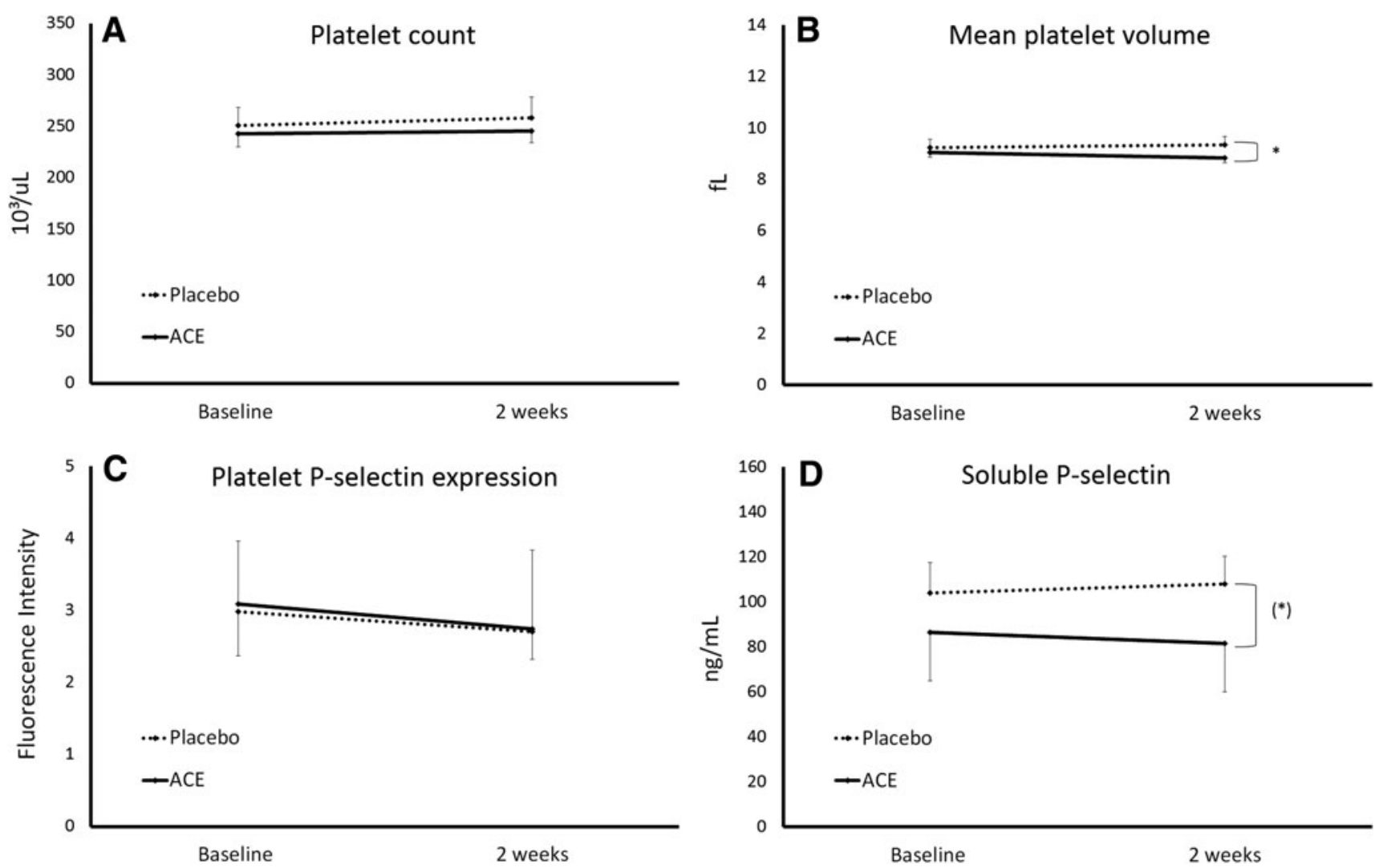

FIG. 2. Parameters pertaining to platelet numbers (A) platelet count, (B) mean platelet volume and activation status, (C) platelet P-selectin expression, and (D) soluble P-selectin are shown as the group averages \pm standard error of the mean. Consumption of ACE was associated with a miniscule but statistically significant reduction in platelet mean volume $\left({ }^{*} P<.02\right)$ and a mild reduction in serum levels of soluble P-selectin $(* P<.06)$. 
Table 5. Blood Clotting Markers

\begin{tabular}{|c|c|c|c|c|c|c|c|c|}
\hline \multirow[b]{2}{*}{ Test (Unit) } & \multirow[b]{2}{*}{ Reference ranges } & \multicolumn{3}{|c|}{ Placebo } & \multicolumn{3}{|c|}{$A C E$} & \multirow[b]{2}{*}{$\mathrm{P}^{* *}$} \\
\hline & & Week 0 & Week 2 & $\mathrm{P}^{*}$ & Week 0 & Week 2 & $\mathrm{P}^{*}$ & \\
\hline aPTT (sec) & $22.7-35.8$ & $25.35 \pm 0.78$ & $25.43 \pm 0.83$ & .896 & $25.30 \pm 0.59$ & $25.78 \pm 0.63$ & .441 & .429 \\
\hline Thrombin time (sec) & $17.2-23$ & $17.87 \pm 0.16$ & $18.57 \pm 0.27$ & .026 & $17.76 \pm 0.12$ & $18.33 \pm 0.24$ & .040 & .695 \\
\hline Fibrinogen activity (mg/dL) & $170-410$ & $311.17 \pm 27.46$ & $294.75 \pm 22.53$ & .127 & $330.75 \pm 16.76$ & $343.67 \pm 24.02$ & .364 & .257 \\
\hline
\end{tabular}

The average \pm standard deviation is shown.

* $P$-values shown for the change from baseline to 2 weeks within each group.

**P-values shown for the difference in the 2 -week change between the two groups.

aPTT, activated partial thromboplastin time.

clotting ability was maintained. Subjective observations by some study participants included one case of increased bruising and three cases of incidents of bleeding gums while consuming ACE. This suggests that daily consumption of high doses of the phycocyanin-rich ACE may have a mild inhibiting effect on the blood clotting system, independent of the clotting factors and markers pertaining to platelet activation included in this study.

A mild but significant reduction in AST and a mild but insignificant reduction in ALT suggested a mild improve- ment in liver function. Even though the AST and ALT enzyme levels remained within the normal range, the reduction may be of some clinical significance. The effect may possibly be due to PCB uptake on digestive breakdown of phycocyanin, as PCB has been reported to protect liver function. ${ }^{37} \mathrm{~A}$ possible mechanism may include the role of Nrf2 in increasing liver enzymes. ${ }^{22}$

Due to an uneven gender representation in the study, gender-specific analysis is limited to the female population. Interestingly, the average percent of activated platelets in
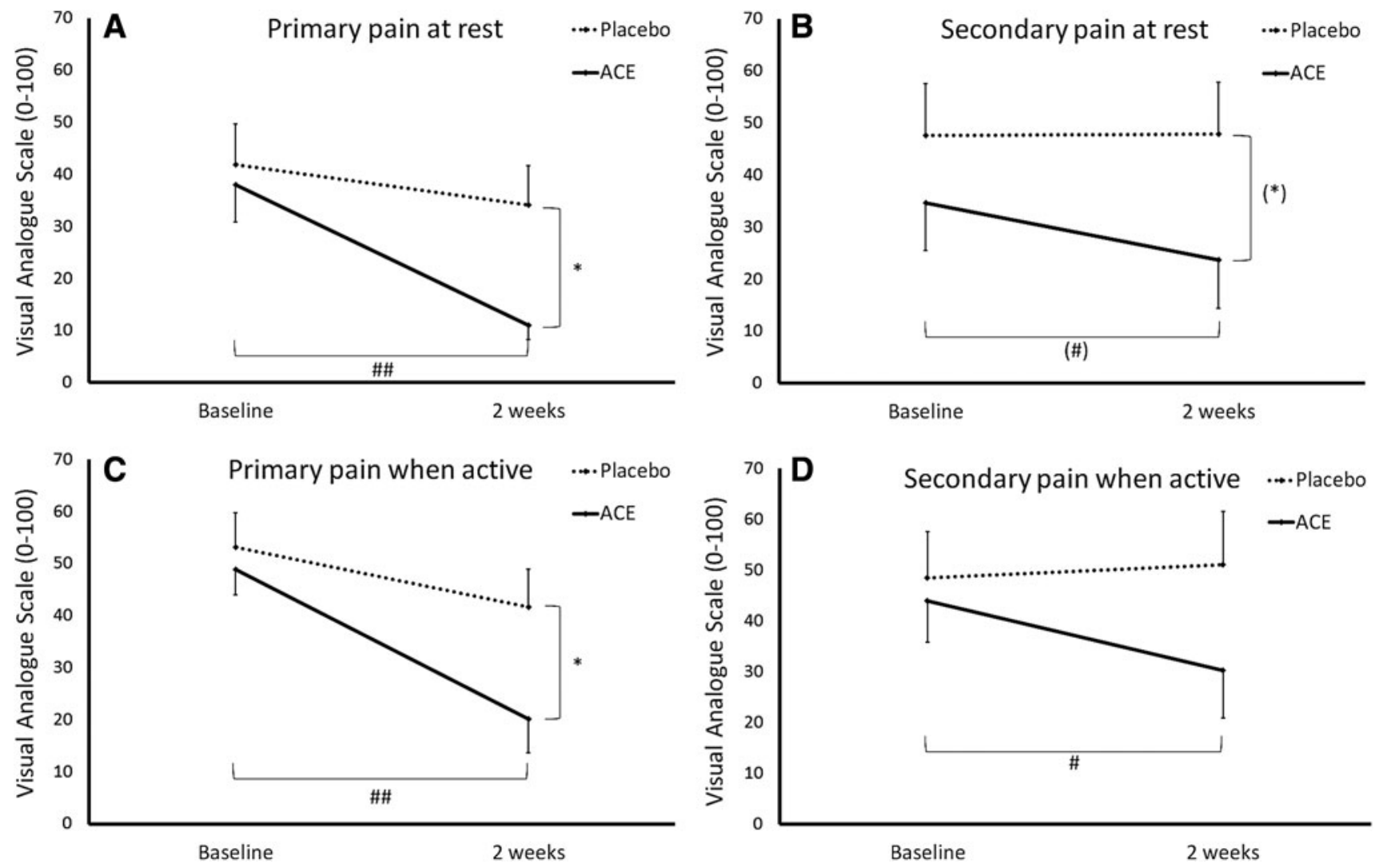

FIG. 3. Pain scores shown as the group averages \pm standard error of the mean for (A) primary and (B) secondary pain complaints at rest and when physically active (C, D). Each individual's anatomical areas for chronic pain were identified before study start, based on the degree with which the pain interfered with daily living. Consumption of the ACE was associated with reduced pain scores. Pain scores for primary pain at rest and when active were similar between the two groups at baseline, but were statistically significant after 2 weeks $(*<.05)$. The reduction in primary pain scores within the ACE group was highly significant $\left({ }^{\# \#} P<.01\right)$. Pain scores for secondary pain at rest showed a statistical trend between the two groups $((*) P<.1)$ as well as within the group consuming ACE $\left(\left(^{\#}\right) P<.1\right)$. The reduction in pain scores for secondary pain when active was statistically significant within the group consuming $\mathrm{ACE}\left({ }^{\#} P<.05\right)$. 
the blood circulation was reduced by $49 \%$ in females consuming ACE, in contrast to only $9 \%$ in the group of females consuming placebo, further suggesting a broad antiinflammatory effect of ACE. The average reduction in soluble P-selectin was seen for both genders and was more robust in the male population consuming ACE (16\% reduction), compared to the female population consuming ACE (8\% reduction).

This study also verified previous documentation of relief of chronic pain associated with consumption of ACE. Based on previous data, ${ }^{32}$ it was not surprising that rapid and robust pain relief was seen for the higher dose used in this study. We suggest that the pain reduction may be due to the complex effects of phycocyanin in inhibiting COX-2 enzymatic activity, as well as PCB effects on reducing NADPH oxidase and increasing HMOX1 activity as the consumed phycocyanin is digested. Furthermore, the anti-inflammatory effects of the nonphycocyanin fraction include inhibition of lipoxygenase activity and additional anti-inflammatory effects, independent of phycocyanin. ${ }^{31}$ It is important that the observed relief of chronic pain was associated with a good safety profile pertaining to blood coagulation. Overall, anti-inflammatory effects, such as effects on C-reactive protein and inflammatory cytokines, were not included in this study.

In conclusion, this report adds substantial weight to the safety portfolio for phycocyanin-containing consumables and extracts. This 2-week study, even though short, was an important step in ongoing safety documentation, and based on the lack of safety concerns in a fairly healthy population, it should now be followed by longer studies. Future studies are warranted to expand on this safety documentation, including studies of longer duration and including premenopausal females. Further studies are also warranted for evaluating safety in the training athlete and other populations that may consume high amounts of phycocyanin-rich extracts to reduce pain and accelerate resolution of exercise-induced inflammation.

\section{ACKNOWLEDGMENTS}

The study was conducted at NIS Laboratory, an independent contract research laboratory specializing in natural products research. The study was sponsored by Cerule LLC, the manufacturer and distributor of the nutraceutical extract tested in this study.

\section{AUTHOR DISCLOSURE STATEMENT}

G.S.J., M.L., and K.F.B. are employees at NIS Laboratory, an independent contract research laboratory specializing in natural products research. C.D. is employed by the study sponsor Cerule LLC.

\section{REFERENCES}

1. Koru E: Earth food Spirulina (Arthrospira): Production and quality standards. In: Food Additive (Yehia El-Samragy, ed.). InTech, Rijeka, Croatia, 2012, pp. 191-202.
2. Palaez F: The historical delivery of antibiotics from microbial natural products - can history repeat? Biochem Pharmacol 2006; 71:981-990.

3. Romay C, Armesto J, Ramerez D, Gonzalez R, Ledon N, Garcia I: Antioxidant and anti-inflammatory properties of C-phycocyanin from blue-green algae. Inflamm Res 1998;47:36-41.

4. Madhava C, Reddya, Subhashinia J, Mahipala SVK, Bhatc VB, Reddya PS, Kiranmala G, Madyastha KM, Reddanna P: CPhycocyanin, a selective cyclooxygenase- 2 inhibitor, induces apoptosis in lipopolysaccharide-stimulated RAW 264.7 macrophages. Biochem Biophys Res Commun 2003;304:385-392.

5. Romay C, Ledon N, Gonzalez R: Further studies on antiinflammatory activity of C-Phycocyanin in some animal modes of inflammation. Inflamm Res 1998;47:334-338.

6. Lanas Á, Carrera-Lasfuentes P, Arguedas Y, García S, Bujanda L, Calvet X, Ponce J, Perez-Aísa Á, Castro M, Muñoz M, Sostres C, García-Rodríguez LA: Risk of upper and lower gastrointestinal bleeding in patients taking nonsteroidal anti-inflammatory drugs, antiplatelet agents, or anticoagulants. Clin Gastroenterol Hepatol 2015;13:906-912.

7. Hsu CC, Wang H, Hsu YH, Chuang SY, Huang YW, Chang YK, Liu JS, Hsiung CA, Tsai HJ: Use of nonsteroidal antiinflammatory drugs and risk of chronic kidney disease in subjects with hypertension: Nationwide longitudinal cohort study. Hypertension 2015;66:524-533.

8. Wang X, Peters U, Potter JD, White E: Association of nonsteroidal anti-inflammatory drugs with colorectal cancer by subgroups in the VITamins and Lifestyle (VITAL) study. Cancer Epidemiol Biomarkers Prev 2015;24:727-735.

9. Bedenis R, Lethaby A, Maxwell H, Acosta S, Prins MH: Antiplatelet agents for preventing thrombosis after peripheral arterial bypass surgery. Cochrane Database Syst Rev 2015;2:CD000535.

10. Su B, O'Connor JP: NSAID therapy effects on healing of bone, tendon, and the enthesis. J Appl Physiol 2013;115:892-899.

11. Albers A, Carli A, Routy B, Harvey EJ, Séguin C: Treatment with acetylsalicylic acid prevents short to mid-term radiographic progression of nontraumatic osteonecrosis of the femoral head: A pilot study. Can J Surg 2015;58:198-205.

12. Konstantinidis I, Papageorgiou SN, Kyrgidis A, Tzellos TG, Kouvelas D: Effect of non-steroidal anti-inflammatory drugs on bone turnover: An evidence-based review. Rev Recent Clin Trials 2013;8:48-60.

13. Hauser RA: The acceleration of articular degeneration in osteoarthritis by nonsteroidal anti-inflammatory drugs. J Prolother 2010;2:305-321.

14. Filaretova LP, Bagaeva TR, Morozova OY, Zelena D: A wider view on gastric erosion: Detailed evaluation of complex somatic and behavioral changes in rats treated with indomethacin at gastric ulcerogenic dose. Endocr Regul 2014;48:163-172.

15. Hougee S, Faber J, Sanders A, Berg WB, Garssen J, Smit HF, Hoijer MA: Selective inhibition of COX-2 by a standardized $\mathrm{CO} 2$ extract of Humulus lupulus in vitro and its activity in a mouse model of zymosan-induced arthritis. Planta Med 2006;72:228-233.

16. Prabhavathi K, Chandra US, Soanker R, Rani PU: A randomized, double blind, placebo controlled, cross over study to evaluate the analgesic activity of Boswellia serrata in healthy volunteers using mechanical pain model. Indian J Pharmacol 2014;46:475-479.

17. Zhu Q, Sun Y, Yun X, Ou Y, Zhang W, Li JX: Antinociceptive effects of curcumin in a rat model of postoperative pain. Sci Rep 2014;4:4932. 
18. Belay A: Mass culture of Spirulina outdoors: The Earthrise Farms Experience. In: Spirulina platensis (Arthrospira) Physiology, Cell Biology and Biotechnology (Vonshak A, ed.). Taylor \& Francis, London, 1997, pp. 131-158.

19. Cevallos CG, Lilia Barron B, Vazquez-Sanchez J: Toxicologic studies and antitoxic properties in Spirulina. In: Spirulina in Human Nutrition and Health (Gershwin ME, Belay A, eds.). CRC Press Taylor \& Francis Group, Boca Raton, FL, 2008, pp. 42-46.

20. Gao Y, Liu C, Wan G, Wang X, Cheng X, Ou Y: Phycocyanin prevents methylglyoxal-induced mitochondrial-dependent apoptosis in INS-1 cells by Nrf2. Food Funct 2016;7:1129-1137.

21. Biswas C, Shah N, Muthu M, La P, Fernando AP, Sengupta S, Yang G, Dennery PA: Nuclear heme oxygenase-1 (HO-1) modulates subcellular distribution and activation of Nrf2, impacting metabolic and anti-oxidant defenses. J Biol Chem 2014;289: 26882-26894.

22. Reuland DJ, Khademi S, Castle CJ, Irwin DC, McCord JM, Miller BF, Hamilton KL. Upregulation of phase II enzymes through phytochemical activation of Nrf2 protects cardiomyocytes against oxidant stress. Free Radic Biol Med 2013;56:102-111.

23. Terry MJ, Maines MD, Lagarias JC: Inactivation of phytochrome- and phycobiliptotein-chromophore precursors by rat liver biliverdin reductase. J Biol Chem 1993;268:26099-26106.

24. Benedetti S, Benvenuti F, Scoglio S, Canestrari F: Oxygen radical absorbance capacity of phycocyanin and phycocyanobilin from the food supplement Aphanizomenon flos-aquae. J Med Food 2010;13:223-227.

25. Marín-Prida J, Pavón-Fuentes N, Llópiz-Arzuaga A, FernándezMassó JR, Delgado-Roche L, Mendoza-Marí Y, Santana SP, Cruz-Ramírez A, Valenzuela-Silva C, Nazábal-Gálvez M, Cintado-Benítez A, Pardo-Andreu GL, Polentarutti N, Riva F, Pentón-Arias E, Pentón-Rol G: Phycocyanobilin promotes PC12 cell survival and modulates immune and inflammatory genes and oxidative stress markers in acute cerebral hypoperfusion in rats. Toxicol Appl Pharmacol 2013;272:49-60.

26. Zheng J, Inoguchi T, Sasaki S, Maeda Y, McCarty MF, Fujii M, Ikeda N, Kobayashi K, Sonoda N, Takayanagi R: Phycocyanin and phycocyanobilin from Spirulina platensis protect against diabetic nephropathy by inhibiting oxidative stress. Am J Physiol Regul Integr Comp Physiol 2013;304:R110-R120.

27. McCarty MF, Barroso-Aranda J, Contreras F: Oral phycocyanobilin may diminish the pathogenicity of activated brain microglia in neurodegenerative disorders. Med Hypotheses 2010; 74:601-605.

28. Pastori D, Pignatelli P, Carnevale R, Violi F: Nox-2 upregulation and platelet activation: Novel insights. Prostaglandins Other Lipid Mediat 2015;120:50-55.

29. Strasky Z, Zemankova L, Nemeckova I, Rathouska J, Wong RJ, Muchova L, Subhanova I, Vanikova J, Vanova K, Vitek L, Nachtigal P: Spirulina platensis and phycocyanobilin activate atheroprotective heme oxygenase-1: A possible implication for atherogenesis. Food Funct 2013;4:1586-1594.

30. Piantadosi CA, Withers CM, Bartz RR, MacGarvey NC, Fu P, Sweeney TE, Welty-Wolf KE, Suliman HB: Heme oxygenase-1 couples activation of mitochondrial biogenesis to anti-inflammatory cytokine expression. J Biol Chem 2011;286:16374-16385.

31. Madhyastha HK, Radha KS, Sugiki M, Omura S, Maruyama M: Purification of c-phycocyanin from Spirulina fusiformis and its effect on the induction of urokinase-type plasminogen activator from calf pulmonary endothelial cells. Phytomedicine 2006;13: 564-569.

32. Hsiao G, Chou PH, Shen MY, Chou DS, Lin CH, Sheu JR: Cphycocyanin, a very potent and novel platelet aggregation inhibitor from Spirulina platensis. J Agric Food Chem 2005;53: 7734-7740.

33. Chiu HF, Yang SP, Kuo YL, Lai YS, Chou TC. Mechanisms involved in the antiplatelet effect of C-phycocyanin. Br J Nutr 2006; 95:435-440.

34. Jensen GS, Attridge VL, Beaman JL, Guthrie J, Ehmann A, Benson KF: Antioxidant and anti-inflammatory properties of an aqueous cyanophyta extract derived from Arthrospira platensis: Contribution to bioactivities by the non-phycocyanin aqueous fraction. J Med Food 2015;18:535-541.

35. Jensen GS, Attridge VL, Carter SG, Guthrie J, Ehmann A, Benson KF: Consumption of an aqueous cyanophyta extract derived from Arthrospira platensis is associated with reduction of chronic pain: Results from two human clinical pilot studies. Nutr Diet Suppl 2016;8:65-70.

36. Keefe DM: "Agency Response Letter GRAS Notice No. GRN000424," FDA ucm335743, December 2012.

37. Liu J, Zhang QY, Yu LM, Liu B, Li MY, Zhu RZ: Phycocyanobilin accelerates liver regeneration and reduces mortality rate in carbon tetrachloride-induced liver injury mice. World J Gastroenterol 2015;21:5465-5472. 